\title{
Uveíte associada à infecção por Trypanosoma evansi em cães no município de Uruguaiana, RS, Brasil
}

\author{
Uveitis associated to the infection by Trypanosoma evansi in dogs from Uruguaiana, RS, Brazil
}

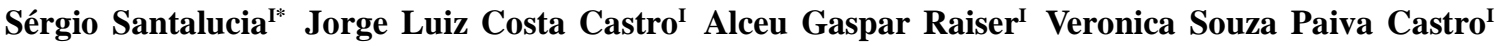 \\ Cintia Ramos Braseiro" ${ }^{\text {II }}$ Luis Felipe Dutra Corrêa ${ }^{\text {III }}$
}

\begin{abstract}
Descrevem-se, neste trabalho, as alterações oculares de dois cães naturalmente infectados por Trypanosoma evansi. Os animais apresentaram, ao exame oftalmológico, teste lacrimal de Schirmer I normal, teste de fluoresceína negativo, quemose, hiperemia conjuntival, secreção mucopurulenta, miose, edema de córnea, pressão intraocular diminuída e efeito Tyndall positivo. No esfregaço sanguíneo, foram identificadas formas tripomastigotas, classificadas como pertencentes à espécie T. evansi. Pelos resultados aqui apresentados, concluímos quanto à necessidade de realização de avaliação oftalmológica completa em cães apresentando uveíte, incluindo exames parasitológicos específicos para hemoparasitas.
\end{abstract}

Palavras-chave: cão, efeito Tyndall, hematozoário, Trypanosoma.

\section{ABSTRACT}

This paper describes the ocular alterations of two dogs naturally infected by Trypanosoma evansi. The animals presented to an ophthalmologic examination, normal Schirmer tear test, negative fluorescein test, chemosis, conjunctival hyperemia, mucopurulent discharge, miosis, corneal edema, intraocular pressure decreased and positive Tyndall effect. In blood smears, trypomastigotes were identified, classified as belonging to the species T. evansi. By the results presented here, it was concluded that there is a necessity of performing a complete ophthalmological examination in dogs with uveitis, including parasitological examination specific to hemoparasites.

Key words: dog, Tyndall effect, hematozoon, Trypanosoma.
Trypanosoma evansi é um protozoário flagelado da seção Salivaria, pertencente à classe Mastigophora e família Trypanosomatidae (JOSHI et al., 2005; COLPO et al., 2005; FRANCISCATO et al., 2007; HOSSEININEJAD et al., 2007), sendo o agente causador da doença conhecido como Mal das Cadeiras em equinos (CONRADO et al., 2005; DOYLE et al., 2007; ZANETTE et al., 2008; Da SILVA et al., 2010). De distribuição geográfica mundial, causa doença significativa em animais de áreas de clima tropical, especialmente África e América Latina (CONRADO et al., 2005), tendo sido descrito infectando equinos, cães, gatos, camelos, bovinos, suínos, búfalos, elefantes, antas, veados, capivaras, quatis, pequenos marsupiais, tatus e o homem (JOSHI et al., 2005; DOYLE et al., 2007; ZANETTE et al., 2008; Da SILVA et al., 2010). Dentre as espécies animais que podem atuar como hospedeiro desse agente infeccioso, HOSSEININEJAD et al. (2007) descreveram o cão como uma das espécies mais afetadas, sendo a equina, de acordo com ZANETTE et al. (2008), a principal espécie afetada em nosso País. No Brasil, a doença causada por T. evansi pode se apresentar na forma aguda, rapidamente fatal em equinos, camelos e cães não tratados (ZANETTE et al., 2008); ou crônica, afetando principalmente os animais silvestres (FRANCISCATO et al., 2007).

\footnotetext{
'Programa de Pós-graduação em Medicina Veterinária, Universidade Federal de Santa Maria (UFSM), Camobi, Santa Maria, RS, Brasil. E-mail: santalucia.sergio@gmail.com. *Autor para correspondência.

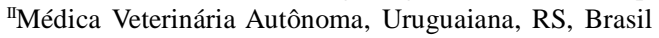

IIIServiço de Oftalmologia Veterinária, Pontifícia Universidade Católica do Rio Grande do Sul (PUCRS), Campus Uruguaiana, Uruguaiana, RS, Brasil.
} 
Os tripanomastigotas presentes no sangue dos vertebrados são transmitidos mecanicamente por moscas hematófagas, dos gêneros Stomoxys sp. e Tabanus sp., e morcegos hematófagos do gênero Desmodus rotundus, quando do repasto sanguíneo (COLPO et al., 2005; CONRADO et al., 2005; ZANETTE et al., 2008; Da SILVA et al., 2010).

Foram atendidos no Serviço de Oftalmologia Veterinária do Hospital Veterinário da Pontifícia Universidade Católica do Rio Grande do Sul (PUCRS) Campus Uruguaiana - dois cães, SRD, sendo um macho e outro fêmea, pesando $8,8 \mathrm{~kg}$ e $15,5 \mathrm{~kg}$, respectivamente, alimentados com comida caseira, provenientes da mesma propriedade, localizada em área rural, com presença de moscas, mosquitos e morcegos. Os cães apresentavam letargia, cansaço, emagrecimento progressivo, inapetência, salivação e diminuição da acuidade visual.

Ao exame físico dos animais, verificou-se infartamento dos linfonodos submandibulares e poplíteos, temperatura $40,3^{\circ} \mathrm{C}$ (macho) e $39,5^{\circ} \mathrm{C}$ (fêmea), presença de estertores pulmonares à auscultação e dermatite descamativa nas orelhas e membros. Ao exame oftalmológico, os cães apresentavam teste lacrimal de Schirmer I normal ${ }^{\text {a }}(19 \mathrm{~mm}$ e $20 \mathrm{~mm})$, quemose, hiperemia conjuntival $(++)$, secreção mucopurulenta, miose, edema de córnea $(++)$, teste de fluoresceína negativo ${ }^{\mathrm{b}}$, pressão intraocular diminuída ${ }^{\mathrm{c}}(8 \mathrm{mmHg})$ e hifema, com humor aquoso turvo (efeito Tyndall positivo) ao exame com lâmpada de fenda ${ }^{\mathrm{d}}$ (Figura $1 \mathrm{~A} \mathrm{e}$ 1B). De posse desses resultados, optou-se pela realização de exames hematológicos como hemograma, perfil bioquímico, pesquisa de hematozoário, esfregaço sanguíneo, além de sorologia para Leishmaniose.

Ao exame hematológico, o macho apresentou pequena alteração no número de eritrócitos (3,3 milhões $\mathrm{mm}^{-3}$ ) e hematócrito (22\%), estando o leucograma $\left(7400 \mathrm{~mm}^{-3}\right)$, proteínas totais $\left(7,2 \mathrm{mg} \mathrm{dl}^{-1}\right)$, creatinina $\left(0,6 \mathrm{mg} \mathrm{dl}^{-1}\right)$ e ALT $\left(97,5 \mathrm{UI} \mathrm{L}^{-1}\right)$ dentro dos valores de normalidade. Ao exame hematológico, a fêmea apresentou pequena alteração nas proteínas totais $\left(7,8 \mathrm{mg} \mathrm{dl}^{-1}\right)$, eritrócitos $\left(3,3\right.$ milhões $\left.\mathrm{mm}^{-3}\right)$ e hematócrito (22\%), estando o leucograma $\left(6400 \mathrm{~mm}^{-3}\right)$, creatinina $\left(1,06 \mathrm{mg} \mathrm{dl}^{-1}\right)$ e ALT $\left(94,2 \mathrm{UI} \mathrm{L}^{-1}\right)$ dentro dos valores de normalidade. $\mathrm{O}$ teste sorológico para leishmaniose realizado em ambos os animais foi reação de imunofluorescência indireta (RIFI), com ambos sendo não reagentes. As formas tripomastigotas observadas no esfregaço sanguíneo (Figura 1C e 1D) e na biópsia aspirativa dos linfonodos foram identificadas, de acordo com dados morfológicos e biométricos, conforme JOHN et al. (1992), como da espécie Trypanosoma evansi.
Após o diagnóstico laboratorial, os animais foram tratados com imizole (duas aplicações com intervalos de 15 dias) e aceturato de diminazeno ${ }^{\mathrm{f}}(3,5 \mathrm{mg}$ $\mathrm{kg}^{-1}$, dose única). Como medicação ocular, os pacientes receberam colírio de acetato de prednisolona $1 \%$, de 8 em 8 horas, e colírio de tobramicina $0,3 \%$, de 4 em 4 horas, ambos durante 14 dias, sendo reavaliados semanalmente até os 30 dias, quando se verificou esfregaço sanguíneo negativo e a regressão total dos sinais oculares. Aos 60 e 90 dias após início do tratamento, os esfregaços sanguíneos continuavam negativos e ausência de sinais oculares.

De acordo com COLPO et al. (2005) e FRANCISCATO et al. (2007), durante a fase aguda da doença, os animais podem apresentar febre intermitente, anemia, edema de membros e partes ventrais do corpo, com emagrecimento progressivo, inapetência, fraqueza e perda de pelos. Acredita-se que ambos os animais do relato apresentavam-se na fase aguda da doença, uma vez que, com exceção do edema em membros e partes ventrais do corpo, todos os demais sinais clínicos de fase aguda foram observados.

A literatura consultada relata que sinais clínicos oculares são observados na fase aguda da tripanossomíase aguda, incluindo conjuntivite, cegueira, lacrimejamento e hifema (CONRADO et al., 2005; HOSSEININEJAD et al., 2007; ZANETTE et al., 2008; Da SILVA et al., 2010), que, embora não sejam citados, são sinais compatíveis com uveíte, corroborando o diagnóstico dessa enfermidade.

Durante a fase subaguda, verificou-se resolução dos sinais clínicos, com a doença não sendo identificada durante um exame físico menos atento, estando o diagnóstico comumente restrito à fase crônica, frente à piora do estado geral do paciente e ao agravamento dos sinais clínicos (COLPO et al., 2005), uma vez que, na fase crônica, os animais infectados apresentam-se debilitados, com mucosas pálidas ou ictéricas, linfonodos superficiais aumentados e incoordenação motora com paralisia dos membros posteriores (ZANETTE et al., 2008; Da SILVAet al., 2010).

Segundo CONRADO et al. (2005), uma vez que os sinais clínicos são inespecíficos, o diagnóstico baseia-se nos achados laboratoriais, consistindo principalmente na pesquisa dos protozoários no sangue, líquido cefalorraquidiano e nodos linfáticos, ou na realização de técnicas diagnósticas como testes sorológicos, culturas sanguíneas e inoculação em animais de laboratório.

Como indicado na literatura, o tratamento consistiu na terapia de suporte e eliminação do protozoário, sendo o aceturato de diminazeno o produto mais comumente utilizado no controle das 


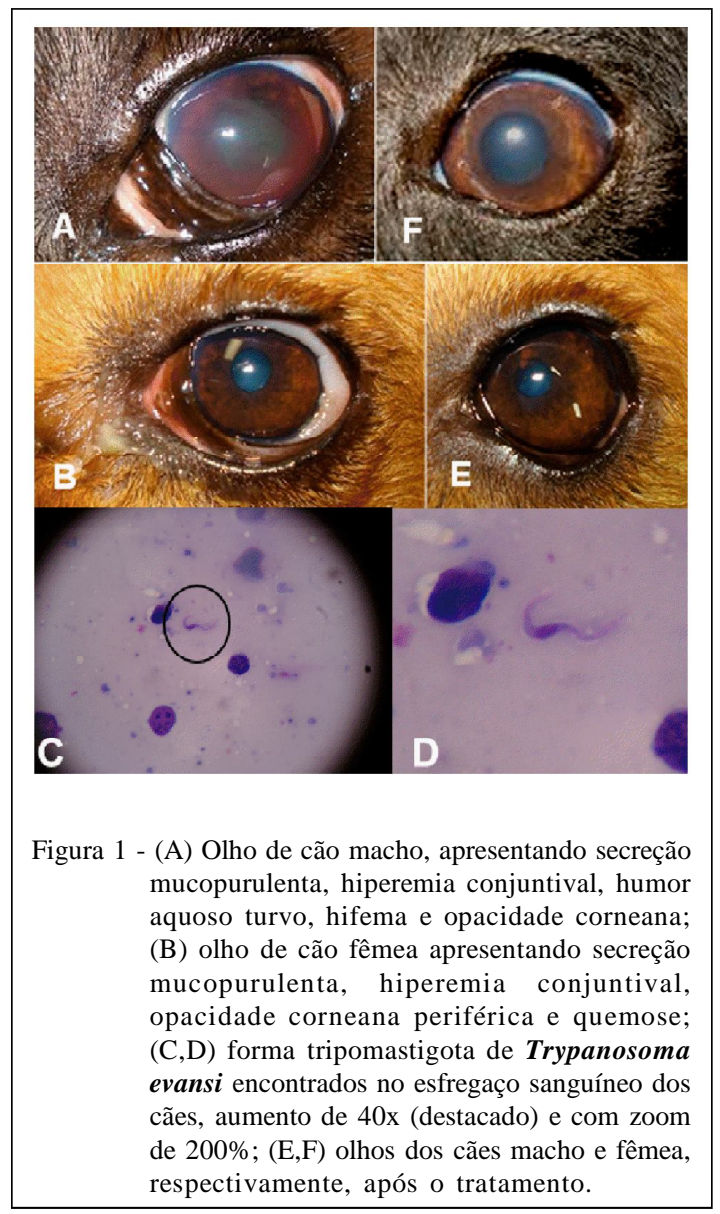

tripanossomíases dos animais domésticos, inclusive tripanossomas resistentes a outros medicamentos (DOYLE et al., 2007; Da SILVA et al., 2010). Embora tenha sido associado com imizole , não é possível afirmar se a associação foi ou não eficaz no tratamento de ambos os animais deste relato, uma vez que a utilização isolada de aceturato de diminazeno se mostra eficaz e também por um estudo experimental realizado por Da SILVA et al. (2008) ter demonstrado que a utilização isolada de imizole $^{\text {e }}$ não é eficaz para o controle da tripanossomose causada por T. evansi.

As uveítes são alterações inflamatórias que afetam toda úvea ou parte dela, tendo como causas mais comuns em cães as doenças virais (ex: cinomose, hepatite infecciosa canina), bacterianas (ex: tuberculose, leptospirose), parasitárias (ex: leishmaniose, toxoplasmose), ou fúngicas (ex: histoplasma, criptococos), além dos traumatismos, tumores e doenças imunomediadas, com os sinais clínicos incluindo dor, fotofobia, miose, lacrimejamento, conjuntivite, edema de córnea e hipotonia ocular. A transparência da câmara anterior pode se modificar frente a um hifema, hipopio e precipitados ceráticos, tornando-se de aspecto turvo (efeito Tyndall positivo) (GELATT \& WILKIE, 2011). O diagnóstico clínico se dá pela observação de um olho vermelho, com injeção ciliar, miose, efeito Tyndall positivo e hipotonia ocular, sendo o tratamento baseado na eliminação do fator etiológico, no controle da dor e no combate à inflamação. Independente da causa, recomenda-se manter o tratamento das uveítes por, no mínimo, seis semanas, em virtude da alta possibilidade de recidiva (SIMON, 2008). Nos casos descritos neste relato, ambos os pacientes do relato receberam medicação tópica para uveíte durante 14 dias, com os sinais oculares regredindo totalmente, não tendo sido considerada necessária a manutenção da medicação por mais de quatro semanas (Figura 1E e 1F).

Os achados aqui descritos destinam-se a alertar a médicos veterinários atuantes na clínica de pequenos animais, quanto à necessidade de inclusão da tripanossomíase por $\boldsymbol{T}$. evansi dentre os possíveis 
diagnósticos diferenciais, quando do atendimento de cães apresentando uveíte, uma vez que a tripanossomose causada por $\boldsymbol{T}$. evansi está cada vez mais presente em diversos estados do Brasil (BRANDÃO et al., 2002; HERRERA etal., 2004; COLPO et al., 2005; CONRADO et al., 2005; FRANCISCATO et al., 2007; ZANETTE et al., 2008).

\section{FONTES DE AQUISIÇÃO}

a - Teste da Lágrima de Schirmer, Ophthalmos Ind. Com. Produtos Farmacêuticos Ltda. Rua das Nhandirobas, 471 Jabaquara - São Paulo, SP, Brasil.

b - Fluoresceína strips, Ophthalmos Ind. Com. Produtos Farmacêuticos Ltda. Rua das Nhandirobas, 471 - Jabaquara São Paulo, SP, Brasil.

c - TonoVet ${ }^{\circledast}$ - Tiolat, Finland.

d - Portable Slit Lamp SL - 15. Kowa Company.

e - Imizol ${ }^{\circledR}$ Injetável, Intervet do Brasil Veterinária Ltda. Rua Cel. Bento Soares, 530, Cruzeiro, SP, Brasil.

f - Beroseg Solução Injetável 7\%, Chemitec Agro-veterinária Ltda. Av. Presidente Wilson, 3458, Ipiranga - São Paulo - SP, Brasil.

\section{REFERÊNCIAS}

BRANDÃO, J.P. et al. Natural infection by Trypanosoma evansi in dog - Case report. Clínica Veterinária, n.36, p.2326, 2002.

COLPO, C.B. et al. Infecção natural por Trypanosoma evansi em cães. Revista Ciência Rural, v.35, n.3, p.717-719, 2005. Disponível em: <http://www.scielo.br/pdf/cr/v35n3/ a38v35n3.pdf>. Acesso em: 30 jan. 2012.

CONRADO, A.C. et al. Infecção natural por Trypanosoma evansi em cavalos na região Central do Estado do Rio Grande do Sul. Ciência Rural, v.35, n.4, p.928-931, 2005. Disponível em: $<$ http://www.scielo.br/scielo.php?script=sci_arttext\&pid=S010384782005000400029>. Acesso em: 27 jan. 2012. doi: 10.1590/ S0103-84782005000400029.

Da SILVA, A.S. et al. Pathological findings associated with experimental infection by Trypanosoma evansi in cats. Journal of Comparative Pathology, v.142, p.170-176, 2010.

Da SILVA, A.S. et al. Aceturato de diminazeno e dipropionato de imidocarb no controle de infecção por Trypanosoma evansi em
Rattus norvegicus infectados experimentalmente. Ciência Rural, v.38, n.5, p.1357-1362, 2008. Disponível em: <http://www.scielo.br/ pdf/cr/v38n5/a25v38n5.pdf>. Acesso em: 25 jan. 2012.

DOYLE, R.L. et al. Eficácia de medicamentos no controle da infecção experimental por Trypanosoma evansi em ratos. Acta Scientiae Veterinariae, v.35, n.1, p.67-71, 2007. Disponível em: 〈http://www.sumarios.org/sites/default/files/pdfs/artigo706.pdf〉. Acesso em: 25 jan. 2012.

FRANCISCATO, C. et al. Cão naturalmente infectado por Trypanosoma evansi em Santa Maria, RS, Brasil. Ciência Rural, v.37, n.1, p.288-291, 2007. Disponível em: <http://www.scielo.br/ pdf/cr/v37n1/a49v37n1.pdf>. Acesso em: 29 jan. 2012.

GELATT, K.N.; WILKIE, D.A. Surgical procedures of the anterior chamber and anterior uvea. In: GELATT, K.N.; GELATT, J.P. Veterinary ophthalmic surgery. Philadelphia: Saunders, 2011. p.237-262.

HERRERA, H.M. et al. Enzootiology of Trypanosoma evansi in Pantanal, Brazil. Veterinary Parasitology, v.125, p.263$275,2004$.

HOSSEININEJAD, M. et al. Trypanosoma evansi in three dogs in Iran. Comparative Clinical Pathology, v.16, p.69$71,2007$.

JOHN, M.C. et al. Biometrical observations on different strains of Trypanosoma evansi. Vet Parasitol, v.43, n.1-2, p.143145, 1992. Disponível em: <http://www.ncbi.nlm.nih.gov/ pubmed/1496797>. Acesso em: 20 maio, 2012.

JOSHI, P.P. et al. Human Trypanosomiasis caused By Trypanosoma evansi in India: the first case report. American Journal of Tropical Medicine and Hygiene, v.73, n.3, p.491495, 2005. Disponível em: <http://www.ncbi.nlm.nih.gov/ pubmed/16172469>. Acesso em: 30 jan. 2012.

SIMON, M. Afecções do trato uveal. In: HERRERA, D. Oftalmologia clínica em animais de companhia. São Paulo: MedVet, 2008. p.173-193.

ZANETTE, R.A. et al. Ocorrência de Trypanosoma evansi em eqüinos no município de Cruz Alta, RS, Brasil. Ciência Rural, v.38, n.5, p.1468-1471, 2008. Disponível em: <http:/ /www.scielo.br/scielo.php?script=sci_arttext\&pid=S010384782008000500045>. Acesso em: 30 jan. 2012. doi: 10.1590/ S0103-84782008000500045. 